\title{
Motivación laboral en los servidores públicos de Ecuador
}

\section{Labor motivation in the public servants of Ecuador}

Ing. María José Vaca Rivas, MBA

Universidad Tecnológica Empresarial de Guayaquil, Ecuador

Autor para correspondencia: mjvaca@uteg.edu.ec

Fecha de recepción: 17 de Abril de 2017 Fecha de aceptación: 30 de Junio de 2017

\section{Resumen}

Los factores motivacionales que influyen en los servidores públicos en el Ecuador, son aspectos de una importancia crucial en el proceso del servicio administrativo público. La gestión de la voluntad de la personas en el trabajo constituye un tema de preocupación en las empresas públicas que conllevan a constructos como la motivación. El objetivo de este artículo es relacionar los factores de motivación laboral que describen el comportamiento de los servidores públicos en la última década, a través del análisis de varias teorías de motivación que permitan determinar los factores que más motivan a un servidor público del país. A partir de los resultados se discuten las consecuencias y la construcción de diseños de modelos teóricos que apuntan finalmente a líneas de investigación futuras.

Palabras Claves: motivación; servidores públicos; sector público; empresa pública

\begin{abstract}
The motivational factors that influence public servants in the Ecuador, are of crucial importance in the process of public administrative service. The management of the will of the people at work is a matter of concern in public organizations underlying constructs as motivation. The aim of this article is to relate the labour motivation factors that describe the behavior of public servants in the past decade, through the analysis of several theories of motivation, determining the factors that motivate more to a public servant in the country. Based on the results, consequences and construction of designs of theoretical models that point finally to future lines of research are discussed.
\end{abstract}

Key words: motivation; civil servants; public sector; public organism. 


\section{Introducción}

Para realizar un análisis es necesario estudiar algunas de las características que son comunes en las organizaciones del sector público, como el conjunto de personas, objetivos, estructura, recursos, contex to o ambiente, resultados y sistema administrativo (Martínez, 1995). Dichas evaluaciones nos permiten establecer diferencias en cuanto al nivel de motivación de los servidores públicos y como puede variar de acuerdo al tipo de organización. De acuerdo a (Etkin, 1994), las empresas públicas tienen características con la interacción con la comunidad, el dialogo, las transacciones y negociaciones con otras instituciones políticas, en su estructura están presentes los afiliados, partidarios, sostenedores, electores y otros tipos de actores sociales. Los empleados públicos se definen como aquellas personas que se encuentran ocupadas en relación de dependencia y cuya actividad se desarrolla en el sector público (Gaspani, Arcidiácono, Carera, Puig, Gluzmann y Brassilo, 2015).

Hernández, Argimon y González-Paramo (2000) exponen que la empresa pública pretende una maximización del bienestar social, pero existen aspectos que hacen difícil la consecución de este propósito, como la multiplicidad de objetivos o un cambio de gobierno, provocando cambios radicales en las directrices e influir sobre la gestión.

El estado tiene un papel importante como empleador, creciendo de manera sostenida durante la última década con un promedio de $12 \%$ de los trabajadores en el sector publico similar al promedio de la región (Gaspani, Arcidiácono, Carera, Puig, Gluzmann y Brassilo, 2015). La actividad de cada una de las empresas públicas, se encuentra diferenciada en su ambiente e influye para determinar algunas relaciones, de este modo, los valores y actitudes establecen las directrices para el comportamiento diario del personal y proporcionan el sentido diferenciador en los servidores públicos de cada empresa. Estos valores se utilizan para establecer los criterios que guían el comportamiento de las personas individualmente, en grupo u organizaciones y tienen que ver con la identificación y motivación de los individuos. La importancia del valor radica en que se convierte en un elemento motivador de las acciones y del comportamiento humano y crea un sentido de identidad del personal con la organización (Denison, 1994).

La motivación debe ser entendida como la trama que sostiene el desarrollo de aquellas actividades que son significativas para la persona y en la que esta toma parte (Ajello, 2003). De esta manera podemos indicar que, la motivación es un aspecto de relevancia en la orientación de acciones y conforma un elemento central que conduce las personas a realizar sus objetivos.

Etimológicamente, el termino motivación procede del latín motus, que se relaciona con aquello que moviliza a la persona para ejecutar una actividad (Trechera, 2005) por lo que es "el conjunto de razones por las que las personas se comportan de las formas en que lo hacen, el comportamiento es vigorosa, dirigido y sostenido" (Santrock, 2002) o su "estado emocional que se genera en una persona como consecuencia de la influencia que ejercen determinados motivos en su comportamiento".(Koenes, 1996).

El estudio se dirige a describir los factores motivacionales de los servidores públicos en el Ecuador y comparar su relación planteándose como objetivos empíricos la descripción y comparación de las posibles diferencias en los factores motivacionales dentro de un grupo de 
empresas públicas. Las diez variables analizadas permiten correlacionar e identificar las necesidades de desarrollo y determinar los criterios para tener en cuenta en los modelos futuros de organización y motivación.

\section{Componente Teórico}

La motivación humana depende de varios factores de estructura y desarrollo, en algunos casos sucede que aunque la necesidad no se satisfaga, tampoco existe frustración, el objetivo se puede transferir a otra necesidad de acuerdo a la situación del individuo, o de la satisfacción de algunas necesidades transitorias. La forma como el motivo se manifiesta depende de cada individuo, su ambiente, su entorno o de la percepción que tenga de un contexto situacional especifico. Para ello existen varias teorías acerca de la motivación, de las cuales se toman en cuenta para este estudio.

\section{Teoría de las necesidades de Maslow}

En su teoría de motivación expuso que las necesidades humanas están jerarquizadas y dispuestas en niveles de acuerdo con su importancia e influencia, las mismas no poseen la misma fuerza o inoperatividad para ser satisfechas y además las ordena en cinco necesidades fundamentales básicas o fisiológicas, de seguridad, sociales, de estima y autorrealización (Maslow, 1943). Esta teoría, una de las más citadas e utilizadas, tiene un gran aporte principalmente el hecho de que "existen en los seres humanos ciertas condiciones internas que tienen la potencialidad de orientar y activar su comportamiento en ciertas direcciones y con cierto grado de intensidad" (Toro y Cabrera, 1981)

\section{Teoría de las relaciones humanas de McGregor}

Establece que "Después de cada decisión o acción gerencial existe una serie de supuestos acerca de la naturaleza del comportamiento humano". (Mac Gregor, 1960). Utilizando como base la jerarquía de necesidades de Maslow, expreso su teoría X y Y que corresponde a dos conceptos opuestos acerca de la naturaleza del hombre. La teoría X establece que el ser humano ordinario siente repugnancia intrínseca hacia el trabajo y lo evitara siempre que pueda, debido a esa tendencia las personas deben ser obligadas a trabajar, controladas, dirigidas y amenazadas con castigos para que desarrollen el esfuerzo adecuado para la realización de los objetivos, su único incentivo es el salario. La teoría Y, por el contrario, propone que la integración de los objetivos individuales con los objetivos de la organización, dependiendo de algunas condiciones, el trabajo es una fuente de satisfacción y no solo acepta sino que busca responsabilidades; una recompensa importante para el individuo es la satisfacción de la necesidad de autorrealización (Koenes, 1996).

\section{Teoría de la motivación - higiene de Frederick Herzberg}

La teoría establecida por Frederick Herzbeg (1959), deriva de dos conjuntos de factores independientes y específicos y explica que existe una relación entre los factores intrínsecos y la satisfacción laboral y entre los factores extrínsecos y la insatisfacción. Los sentimientos de satisfacción, intrínsecos o motivadores se experimentan en el desempeño de sus labores como la 
autorrealización, reconocimiento, progreso, desarrollo profesional y responsabilidad; mientras que los factores de higiene o extrínsecos, que si están presente no originan motivación pero evitan la insatisfacción, son tales aspectos como la relaciones interpersonales, las condiciones físicas de trabajo, la remuneración, seguridad laboral y políticas administrativas de la empresa, (Plumise, 1991).

\section{Teoría de la satisfacción de las necesidades de Mc Clelland}

Mc Clelland (1967) presentó una teoría acerca de la motivación humana enfocada en tres necesidades importantes que ayudan a explicar la motivación: Logro, Poder y Afiliación. El logro es una necesidad de realización que impulsa a los individuos a desempeñar un papel activo en la determinación del resultado y fomenta la fabricación de sus propias oportunidades. Una necesidad de poder se manifiesta por medio de las acciones que buscan ejercer dominio y control, estas personas quieren modificar los comportamientos de los individuos, tener influencias y control sobre los demás, y la necesidad de afiliación, que se infiere por los comportamientos que se encaminan a obtener, conservar y restaurar una relación afectiva con una persona, a mantener relaciones amigables y estrechas y a preferir situaciones donde haya cooperación y ausencia de competencia (Schein, 1991).

Este enfoque facilito la comprensión de algunas características del comportamiento humano, por lo que se han realizado hipótesis acerca de porque unas personas desempeñan mejor que otras su trabajo, porque unas establecen relaciones de dirección y control o relaciones amistosas; estas conductas tienen efectos importantes en la productividad laboral (Toro y Cabrera, 1981).

\section{Teoría de las expectativas}

El estudio de la motivación de Victor H. Vroom (1964) postula que los individuos tienen creencias y expectativas con respecto a su futuro a partir de tres factores los objetivos individuales o la fuerza de voluntad para alcanzar objetivos (expectativas), la relación que el individuo percibe entre la productividad y el logro de sus objetivos individuales (recompensa) y la capacidad del individuo para influir en su nivel de productividad en la medida en que cree poder hacerlo (relaciones entre expectativas y recompensas). Este modelo permite entender fácilmente el proceso mental utilizado para la motivación, es decir, debe tener claro qué tipo de comportamiento predice y en que situaciones se aplica.

Otras teorías expuestas por Luthans y Stajkavic (2001) identifican conductas en los empleados basada en el rendimiento, donde afirman que los incentivos tienen diferentes efectos según su utilidad. Mientras que Adams (1965) considera que la equidad es el trato justo del valor de las personas lo que provoca la motivación para mantener las relaciones con las organizaciones y sus compañeros de trabajo. Carrell y Dittrich (1978) sostiene que el individuo se motiva bastante si percibe la proporción de sus aportaciones frente a los resultados equivalentes de quienes lo rodean.

Para efectos de este estudio y de acuerdo a las teorías antes mencionadas se determinaron los siguientes factores de motivación como modelo de evaluación y medición en los servidores 
públicos: Remuneración, Ambiente Físico, Seguridad, Necesidad de Logro, Reconocimiento, Poder, Expectativa, Equidad, Relaciones Personales y Afiliación.

\section{Metodología}

\begin{tabular}{|l|l|l|l|l|}
\hline $\begin{array}{l}\text { Valor de trabajo } \\
\text { extrínseco }\end{array}$ & $\begin{array}{l}\text { Valor de } \\
\text { trabajo mixto }\end{array}$ & $\begin{array}{l}\text { Valores de } \\
\text { trabajo } \\
\text { intrínsecos }\end{array}$ & $\begin{array}{l}\text { Valores de } \\
\text { trabajo social }\end{array}$ & $\begin{array}{l}\text { Valores de } \\
\text { prestigio }\end{array}$ \\
\hline Remuneración & Expectativas & $\begin{array}{l}\text { Necesidad de } \\
\text { Logro }\end{array}$ & $\begin{array}{l}\text { Relaciones } \\
\text { Personales }\end{array}$ & Reconocimiento \\
\hline Seguridad & Equidad & Afiliación & & Poder \\
\hline Ambiente Físico & & & & \\
\hline
\end{tabular}

Gráfico $\mathrm{N}^{0}$ 1: Tipos de valor de trabajo

Fuente: Elaborado con base a Schwarts ,1992.

Para realizar este estudio se utilizó el método descriptivo, muy utilizado en las ciencias administrativas. La descripción es considerada un subproducto de la observación que presenta las hechos como ocurren, los agrupa y convierte en información (Méndez, 1995), utilizando encuestas que se basan en recoger o recopilar información mediante la aplicación de un cuestionario para conocer las motivaciones, Angura y Arnau (1995) en cada servidor público, seleccionando una muestra entre empresas públicas y servidores públicos diferentes tiempos de servicio para determinar la interrelación de las variables y hacer inferencias a partir de la muestra sobre la población. En base a esto, la investigación es descriptivo correlacional donde la exploración de los distintos modos en los cuales las prioridades de valor de los individuos se relacionan con sus actitudes, su comportamiento, roles y experiencias (Schwarts, Surkiss y Ross, 1999). De esta manera nos proporciona los factores motivacionales en los empleados públicos por tipo de valor de trabajo durante la última década.

\section{Resultados}

El análisis parte del comparativo realizado de la muestra, a través de las encuestas utilizando una escala de Likert donde se multiplica por la intensidad del factor y divido por el número de encuestas realizadas, para obtener de esta manera el promedio de importancia, siendo el menor promedio el que obtiene la mayor prioridad, de esta forma nos permite priorizar los factores motivacionales dando como resultado, la siguiente jerarquización de factores.

\begin{tabular}{|l|c|}
\cline { 2 - 2 } \multicolumn{1}{c|}{} & PROMTOR \\
\hline AFILIACION & 1,84 \\
\hline NECESIDAD DE LOGRO & 2,02 \\
\hline RECONOCIMIENTO & 2,13 \\
\hline AMBIENTE FISICO & 2,23 \\
\hline EXPECTATIVAS & 2,44 \\
\hline SEGURIDAD & 2,47 \\
\hline EQUIDAD & 2,48 \\
\hline PODER & 2,66 \\
\hline REMUNERACION & 2,70 \\
\hline R. PERSONALES & 3,13 \\
\hline
\end{tabular}

Gráfico $\mathbf{N}^{\circ}$ 2: Jerarquización de factores

Fuente: creación de la autora 
Estos resultados fueron contrastados con las entrevistas a profundidad realizada a toda la muestra, de ellos podemos determinar que los factores más motivantes en el sector público en las empresas públicas en Ecuador son la Afiliación como primer lugar, que se define como el deseo de asociación y pertenencia, la Necesidad de Logro que se refiere a los objetivos propuestos que impulsa un mejor rendimiento, el logro de las aspiraciones y sentido de servir a los demás, cumpliendo con las expectativas de los usuarios o ciudadanos; en tercer lugar el Reconocimiento, que es la valoración por parte de sus superiores, compañeros de trabajo y demás personal de la empresa pública; el Ambiente Físico, que se determinó como las condiciones físicas en su lugar de trabajo; Expectativas que se considera como la motivación de una persona para realizar una acción y las esperanza de lograrla; Seguridad, es el sexto factor, que se determina por la permanencia dentro de la organización, su estabilidad en un área de trabajo con políticas y reglamentos claros; El séptimo factor de motivación es la Equidad que se experimenta y satisface cuando lo que recibe está de acuerdo con el esfuerzo realizado; Poder es la capacidad o el potencial de influir sobre los demás y/o tener control sobre algunos medios para cumplir los objetivos de la organización; El noveno factor determinado es la Remuneración que no es otra cosa que el salario, prestaciones sociales o bonificaciones recibidas por el servidor público y por ultimo esta las Relaciones Personales que implican las buenas relaciones personales y sociales con superiores y/o compañeros dentro del área de trabajo.

El análisis de correlación de los factores nos describe el grado o fuerza con que se produce la relación de los factores motivaciones, se utilizó la medida del coeficiente de correlación o correlación Tau_b de Kendal que es apropiada para variables ordinales, dando como resultado la siguiente tabla:

\begin{tabular}{|c|c|c|c|c|c|c|c|c|c|}
\hline & $\begin{array}{c}\text { Ambiente } \\
\text { Físico }\end{array}$ & Seguridad & $\begin{array}{c}\text { Necesidad } \\
\text { de Logro }\end{array}$ & Reconocimiento & Poder & Expectativas & Equidad & $\begin{array}{l}\text { Relaciones } \\
\text { Personales }\end{array}$ & Afiliacion \\
\hline Remuneracion & \multirow[t]{11}{*}{0,37} & 0,371 & 0,453 & 0,382 & 0,229 & 0,247 & 0,225 & 0,088 & 0,197 \\
\hline Ambiente Físico & & \multirow[t]{10}{*}{0,22} & 0,492 & 0,215 & 0,004 & 0,178 & 0,389 & $-0,188$ & 0,133 \\
\hline Seguridad & & & \multirow[t]{9}{*}{0,33} & 0,434 & 0,25 & 0,107 & 0,332 & 0,122 & 0,267 \\
\hline Necesidad de & & & & \multirow{8}{*}{0,424} & & & & & \\
\hline Logro & & & & & 0,301 & 0,408 & 0,556 & 0,085 & 0,512 \\
\hline Reconocimeinto & & & & & \multirow[t]{6}{*}{0,43} & 0,35 & 0,301 & 0,161 & 0,323 \\
\hline Poder & & & & & & \multirow[t]{5}{*}{0,178} & 0,237 & 0,195 & 0,311 \\
\hline Expectativas & & & & & & & 0,522 & 0,147 & 0,415 \\
\hline Equidad & & & & & & & & 0,245 & 0,462 \\
\hline Relaciones & & & & & & & & & \\
\hline Personales & & & & & & & & & 0,149 \\
\hline
\end{tabular}

Gráfico No 3: Correlación de Factores de Motivación Fuente: creación de la autora

Del gráfico anterior, podemos determinar que todas las variables están correlacionadas positivamente una en mayor grado que otras, menos las variables Relaciones Personales y Ambiente Físico, la misma que tiene una correlación negativa muy baja.

\section{Discusión y Conclusiones}

Los resultados obtenidos de este estudio nos permiten determinar que los factores más motivantes dentro de los servidores públicos en el Ecuador son la Afiliación, Necesidades de Logro y el Reconocimiento, que abren interesantes debates sobre la relación a otros factores y su grado de importancia. 
Empezare señalando que de acuerdo a la Ley Orgánica de Servicio Público (LOSEP,2010) donde se plantea que el servicio público y la carrera administrativa tienen por objetivo propender el desarrollo profesional, técnico y personal de los servidores públicos para lograr el mejoramiento, eficiencia, eficacia, calidad, productividad del Estado y de sus instituciones, mediante la conformación del funcionamiento y desarrollo de un sistema de gestión del talento humano sustentado en la igualdad de derechos, oportunidades y la no discriminación, lo que buscó institucionalizar las entidades rectoras del sistema del servicio civil estableciendo parámetros tanto de contratación, remuneración y evaluación de los servidores públicos, de esta manera permite los cambios que revela el estudio de diagnóstico institucional del servicio civil (BID, 2014), donde indica que se ha pasado de una situación de debilidad entre tres factores fundamentales como la equidad salaria, la carrera administrativa y el incentivo no remunerado como la formación de factores de desmotivación y están reflejados en los cambios establecidos como los concursos abiertos de oposición y merito, elaboración de manuales de puestos, la homologación salarial con mínimos y máximos, el actual trabajo sobre incentivos monetarios, el desarrollo de un sistema de información integral y el fortalecimiento del sistema de gobierno del servicio civil. Esto genera que los factores motivacionales hayan cambiado durante la última década, y actualmente los factores motivantes como remuneración, poder y relaciones personales no dejan de presentar un reto interesante para la gestión de motivación en el sector público, sin embargo son factores que motivan de cierta manera si están presentes con un impacto menor que los demás porque suplen una necesidad momentánea. Además el sistema de contratación actual establecido por la ley de Servicio Orgánico de Servicio Público permite a los empleados públicos cuenten con una mayor estabilidad siendo este un factor importante pero no predominante. Sin embargo, esto amplia los debates hacia los factores de mayor motivación como la Afiliación, Necesidad de Logro y Reconocimiento, para perfeccionar los instrumentos de medición y aplicación de técnicas de análisis más poderosas y construir una modelo de escala libre de sesgos, por eso se hace imperativo fomentar esfuerzos para promover procesos de evaluación y diagnósticos para enriquecer la afiliación, la necesidad de logro y reconocimiento entre los empleados públicos que son la base para la construcción de una sociedad basada en la solidaridad, justicia social, la igualdad de oportunidades.

\section{Bibliografía}

Ajello, A.M. (2003). La motivación para aprender. En C. Pontecorvo (Coord.), Manual de Psicología de la educación (pp. 251-271) España: Popular.

Angura, M. \& Arnau, J. (1995). Métodos de investigación en psicología. España: editorial Síntesis.

BID (2014). Banco Interamericano de Desarrollo. Diagnóstico Institucional del Servicio Civil en América Latina: Ecuador.

Carrell, M., \& Dittrich, J. (1978). Equity Theory: The Recent Literature, Methodological consideration, and New Directions. The Academy of Management Review, 2,202-210.

Denison, D. (1994). Cultura Corporativa. Bogotá: Legis.

Etkin, J. (1994).La doble moral de las organizaciones. España: MacGraw-Hill. 
Gasparini, L., Arcidiácono, M., Carella, L., Puig, J., Gluzmann, P., \& Brassiolo, P. (2015). EL Empleo Público En América Latina: Evidencia de las encuestas de hogares *. El Trimestre Económico, 82(4), 749-784.

Hernández, P., Argimón, I. \& González-Paramo, J.M (2000). ¿Afecta la titularidad pública la eficiencia empresarial? España: Imprenta del Banco de España.

Herzberg, F., Mausber, B. \& Snyderman, B. (1959) The motivation to work. Nueva York: Jhon Wiley \& Sons Inc.

Koenes, A. (1996). Gestión y motivación del personal. España: Ediciones Diaz de Santos.

LOSEP (2010).Ley Orgánica de servicio Público. Registro Oficial Suplemnto 294 del 06-Octubre del 2010. Última modificación 28 de Marzo 2016.

Luthans, F. \& Stajkavic, A. (2001). Differential effect on incentive motivators on work performance. Academy of management journals, 4, 580-582.

Maslow, A. (1943). A theory of Human Motivation. Psychological Review, 50, 96-370

Mendez, Carlos (1995). Metodologia. Mexico: McGraw-Hill.

McClelland, D. (1967). The Achieving Society. New York.

McGregor, D. (1960). The human side of Enterprises (D. McGregor Ed.). New York: Editorial McGraw Hill.

Martínez, C., Cabuya, L. et al. (1995).Organización y Gestión de Empresas. Bogotá: Nomos S.A.

Plumise, L. (1991). A Visit with Fred Herzberg. Management Newsletter, 27.

Santrock, J. (2002). Psicología de la educación. México: Mac Graw-Hill.

Schein, E. (1991) Psicología de la organización. México: Pretince-Hall.

Schwartz, S. (1992). Universals in the content and structure of values: Theory and empirical test in 20 countries. En M. Zanna (Ed.), Advances in experimental social psychology (pp.1-65). New York, NY: Academic Press.

Schwartz, S., Surkiss, S \& Ros, M. (1999). Valores Básicos Individuales, Valores de Trabajo y Significado de Trabajo. Psicología aplicada; Revisión internacional 48(1), 49-71

Toro, F. \& Cabrera, H. (1981). Motivación para el trabajo. Medellín: Ediciones Gráficas.

Trechera, J. L. (2005). Saber motivar: ¿El palo o la zanahoria?

Vroom, V. (1964). Work and Motivation New York: Academic Press. 